swimming training on serum free fatty acid (FFA) and the expression of peroxisome proliferator-activated receptor (PPAR- $\gamma$ ), carnitine palmitoy1 transferase-1 (CPT-I), medium-chain acy1-coenzyme A dehydrogenase (MCAD) mRNA, to explore the mechanisms that Why swimming training could improve insulin resistance.

Methods Twenty-six male ApoE knockout mice were randomly divided into groups: the high-fat diet group (HFD, $n=13$ ) and the high-fat diet group with exercise training (HFD+Ex, $n=13)$. The HFD+Ex group were fed with high-fat diet with exercise training for 12 weeks. The treatment of HFD group was identical to the HFD+Ex group except to swimming training. And another ten healthy male C57BL/6J (ND, $\mathrm{n}=10$ ) mice as the control group were, which were fed with normal diet for 12 weeks. After 12 weeks experiment, the mice were dissected, the livers were excided off immediately. Serum insulin, glucose were determined and Homa-IRI was calculated to ascertain the establishment of insulin resistance. Serum total cholesterol (TC), trigiyceride (TG), high-density lipoprotein cholesterol (HDL), low-density lipoprotein cholesterol (LDL), free fatty acid (FFA) were determined. The PPAR- $\gamma$, CPT-I, MCAD mRNA in liver were estimated by reverse transcription PCR (RT-PCR).

Results 1. Compared with the ND group, the body weight of the HFD group was significantly higher $(\mathrm{p}<0.05)$. Compared to the HFD group, the body weight of the HFD+Ex group was significantly lower $(p<0.05)$. 2. Compared with the ND group, fasting glucose, insulin and Homa-IRI of the HFD group was significantly higher $(p<0.01)$. Compared with the HFD group, fasting insulin, glucose and HOMA-IR of the HFD+Ex group was significantly lower $(p<0.05,0.01,0.01)$. 3. Compared with the ND group, TC, LDL, FFA of HFD group was significantly higher $(p<0.01)$, TC, LDL of the HFD+Ex group was still higher $(p<0.01)$. Compared with the HFD group, TC, LDL, FFA of the HFD+Ex group was significantly lower $(p<0.05,0.05,0.01)$, HDL was significantly higher $(p<0.05)$. 4. Compared with the ND group, the expression of PPAR- $\gamma$, CPT- 1 , MCAD mRNA of the HFD group were significantly deceased $(p<0.01)$; Compared with the HFD group, the expression of PPAR$\gamma$, CPT-1, MCAD mRNA of the HFD group were significantly increased $(p<0.01)$.

Conclusion 1. High-fat diet could induce insulin resistance of ApoE Knockout mice. 2. Swimming training could improve insulin resistance of ApoE knockout mice. 3. Swimming training could improve insulin resistance possibly through upregulating the expression of PPAR- $\gamma$, CPT-1, MCAD mRNA.

Funding This study is supported by Natural Sciences Foundation of Hunan. N0.06SK3090.

\section{e0281 RELATIONSHIP BETWEEN ALBUMINURIA AND BLOOD PRESSURE LEVEL IN PATIENTS WITH ESSENTIAL HYPERTENSION}

doi:10.1136/hrt.2010.208967.281

\begin{abstract}
${ }^{1}$ Sun Ningling, ${ }^{1}$ Wang Hongyi, ${ }^{2}$ Zhu Dingliang, ${ }^{3}$ Liao Yuhua, ${ }^{4}$ Lin Shuguang, ${ }^{5}$ Chen Xiaoping. ${ }^{1}$ People's Hospital Peking Universtiy; ${ }^{2}$ Hypertension Research Institute Shanghai; ${ }^{3}$ Union Hospital Wuhan; ${ }^{4}$ Guangdong General Hospital; ${ }^{5}$ Sichun University Westchina Hospital
\end{abstract}

Objective To investigate the relationship between albuminuria detection rate and blood pressure level or body weight index (BMI) in patients with essential hypertension in China's five regions.

Method 5021 patients with clearly diagnosed essential hypertension but without diabetes were enrolled in our study. The participants came from five cities of China. Urinary albumin/creatinine ratio was measured in these patients for two times. The relationships between albuminuria and levels of blood pressure and BMI were analysed.

Results 1 . There wasn't any difference of the albuminuria incidence between patients less than 60-year-old and equal to or more than
60 -year-old. The longer the hypertension exists, the higher the proportion of albuminuria. 2. The incidence of albuminuria associated with blood pressure levels significantly. The higher the blood pressure (BP) level, the more the urine protein excretion. The albuminuria detection rate in patients with normal blood pressure, high normal blood pressure, I, II or III stage hypertension were $26.3 \%$, $27.3 \%, 28.7 \%, 31.5 \%$ and $40.3 \%$ respectively. 3 . The incidence of albuminuria was different in patients with uncontrolled BP (BP $>=140 / 90 \mathrm{~mm} \mathrm{Hg}$ ) compared with those with well controlled $\mathrm{BP}(<140 / 90 \mathrm{~mm} \mathrm{Hg})(27.1 \%$ vs $30.2 \%, \mathrm{p}<0.05)$. 4. The incidence of albuminuria was in obese patients compared with those with normal body weight at equal blood pressure level, but the difference wasn't statistically significant. 5. Patients with albuminuria had more heart, cerebral or renal events than whom without proteinuria. Conclusion The detection rate of proteinuria in hypertensive patients without known diabetes was $28.8 \%$ in China, among them the incidence of microalbuminuria was $18.6 \%$, and the incidence of clinical albuminuria was $10.2 \%$. Uncontrolled BP was important risk factor of proteinuria.

\section{E0282 EFFECT OF COMPREHENSIVE INTERVENTION ON ANXIETY AND DEPRESSIVE SYMPTOMS IN PATIENTS AFTER ACS}

doi:10.1136/hrt.2010.208967.282

${ }^{1}$ Liu Guanghui, ${ }^{1}$ Wu Xianzheng, ${ }^{2}$ Ma Wenlin, ${ }^{1}$ Su Lijie, ${ }^{3} \mathrm{Hu}$ Dayi. ${ }^{1}$ Emergency Internal Medicine, Tongji Hospital, Tongji University; ${ }^{2}$ Department of Cardiology, Tongji Hospital, Tongji University; ${ }^{3}$ Heart Center Peking University Peoples Hospital

Objective To evaluate the effect of comprehensive intervention to the prognosis of depressive and/or anxiety symptoms in future. To provide a reasonable and reliable intervention instrument to treat ACS patients.

Methods To select acute coronary syndrome with anxious and/or depressive symptoms patients 268 examples, all patients were divided into the intervention group 134 examples and the control group 134 examples. On the base of conventional therapy, Patients of intervention group received comprehensive intervention (health education, exercise, psychological relaxation et al). Before the intervention and after follow-up test the HADS, to follow up with the patients to observe the dynamic change of emotion. The state of anxiety and depression in all patients were analysed according to psychological test scale.

Results 1. Total 256 example patient include the intervention group $(n=130)$ and the control group $(n=126)$, after giving the treatment has carried on at least two times evaluation. Because loses the revisit 6 examples, died 4 examples, uncompliant patients 6 examples, completed finally experiments 240 examples. 2. Comparing two group's emotion in the two group patients respectively at baseline and after intervention. The HADS scores carried on the difference examination separately: Before the comprehensive intervention, the intervention group's HAD-a, HAD-d, HAD-t are 8.92 $\pm 3.72,8.73 \pm 3.41,17.68 \pm 3.72$ respectively, the control group's scores are 8.67 $\pm 3.13,8.52 \pm 3.06,17.47 \pm 3.27$ respectively, $\mathrm{p}>0.05$; After intervention, for the invention group, HAD-a, HAD-d, HAD-t are 5.82 $\pm 1.41,6.84 \pm 1.86,11.93 \pm 1.59$; The control group's are $6.76 \pm 1.31,7.28 \pm 1.58,13.62 \pm 1.36$ respectively, $\mathrm{p}<0.05$.

Conclusions For the intervention group patients, comprehensive intervention (Coronary heart disease treatment, Health education, psychological counseling, Relaxation training, taking exercise et al) may obviously alleviate the anxiety and/or depressive symptoms in ACS patients, it is useful to the prognosis improvement of QOL. 2. On the basis of medication for ACS, for the control group patients, the anxiety and/or depressive symptom has been alleviated to some extent. 